\title{
Varianzschätzung von Nettoveränderungen mit dem Mikrozensus ab 2012
}

\author{
Bernhard Schimpl-Neimanns
}

Eingegangen: 11. Juli 2018 / Angenommen: 11. März 2019 / Online publiziert: 20. März 2019

(C) Der/die Autor(en) 2019

Zusammenfassung In diesem Aufsatz wird die Varianzschätzung von Nettoveränderungen am Beispiel ausgewählter Indikatoren des Mikrozensus untersucht. Auf der Grundlage von Scientific-Use-Files des Mikrozensus als große replikative Mehrthemenumfrage werden häufig zeitliche Veränderungen von Indikatoren berichtet. Durch die partielle Rotation von Erhebungseinheiten reduziert sich die Varianz von Nettoveränderungen. Dieser methodische Vorteil kann seit der Bereitstellung längsschnittkonsistenter Ordnungsnummern in den Daten ab 2012 genutzt werden. Hierfür wird das von Berger und Priam (2016) vorgeschlagene Verfahren verwendet. Damit ist es nun für die Wissenschaft möglich, die hohe Präzision der Schätzergebnisse von Nettoveränderungen effizient auszuschöpfen.

Schlüsselwörter Mikrozensus · Rotationspanel · Varianzschätzung ·

Nettoveränderungen

\section{Variance estimation of net changes with the German Microcensus since 2012}

Abstract This article examines the variance estimation of net changes using selected indicators of the Microcensus as an example. Based on the scientific use files of the Microcensus, which is a large replicative multi-topic survey, temporal changes of indicators are often reported. The partial rotation of survey units reduces the variance of net changes. This methodological advantage can be used since the provision of longitudinally consistent identification numbers in the data from 2012. The procedure proposed by Berger and Priam (2016) will be applied for this pur-

B. Schimpl-Neimanns $(\triangle)$

GESIS - Leibniz-Institut für Sozialwissenschaften, B2, 1, 68159 Mannheim, Deutschland

E-Mail: bernhard.schimpl-neimanns@gesis.org 
pose. This makes it possible for the scientific community to make efficient use of the high precision of the estimation results of net changes.

Keywords German Microcensus · Rotation Panel · Variance Estimation · Net Change

\section{Einleitung}

Aufgrund des großen Stichprobenumfangs und des jährlichen Erhebungszyklus bietet der Mikrozensus (MZ) für die Forschung einige Vorteile für Analysen des sozialen und wirtschaftlichen Wandels. Für Vergleiche von Merkmalsveränderungen von Indikatoren oder Merkmalswerten auf aggregierter Ebene zwischen Zeitpunkten (Nettoveränderungen) in der Form von Trendstudien oder der Analyse replikativer Surveys stehen der Wissenschaft Scientific-Use-Files (SUF) als 70\%-Substichproben des MZ zur Verfügung. Mit der Bereitstellung längsschnittkonsistenter Ordnungsnummern ab dem SUF des MZ 2012 ist es möglich geworden, bei der Schätzung der Varianz der Nettoveränderung die durch die partielle Rotation der Primäreinheiten entstehende Kovarianz der Indikatoren im Zeitverlauf zu schätzen. Der Beitrag beschreibt die neuen Analysemöglichkeiten am Beispiel ausgewählter Indikatoren und wendet das von Berger und Priam (2016) vorgeschlagene Verfahren an. Zugleich wird gezeigt, welche Fehler entstehen, wenn eine nicht zutreffende statistische Unabhängigkeit der Teilstichproben angenommen wird.

Der Beitrag ist wie folgt aufgebaut. Zunächst werden das Erhebungsdesign des Mikrozensus und die Ziehung der Substichprobe des SUF zusammenfassend beschrieben (Abschn. 2). Im dritten Abschnitt wird zuerst skizziert, wie designbasierte Schätzungen für Querschnittsdaten bei gebundener Hochrechnung mittels Regressionsschätzung nach dem Linearisierungsansatz vorgenommen werden können. Anschließend wird die Varianzschätzung für Nettoveränderungen vorgestellt. Beispielhafte Analysen folgen im Abschn. 4. Der Beitrag schließt mit einer Zusammenfassung.

\section{Datenbeschreibung}

Der MZ wird jährlich als geschichtete einstufige Klumpenstichprobe mit einem Auswahlsatz von $1 \%$ erhoben. Eine partielle Rotation der Erhebungseinheiten unterstützt möglichst präzise Schätzungen sowohl für jeden Erhebungszeitpunkt als auch für Vergleiche zwischen Zeitpunkten. Die Auswahlbezirke und die darin wohnenden Haushalte und Personen verbleiben vier Jahre lang in der Befragung und bilden ein sogenanntes Rotationsviertel. Jährlich wird ein Viertel der Auswahlbezirke ausgetauscht (siehe Abb. 1). Populationsveränderungen, die beispielsweise durch Fortund Zuzüge, Geburten und Sterbefälle entstehen, werden dadurch automatisch erfasst. Betrachtet man zwei aufeinanderfolgende Jahre, sind $75 \%$ der Auswahlbezirke in beiden Stichproben vertreten. 


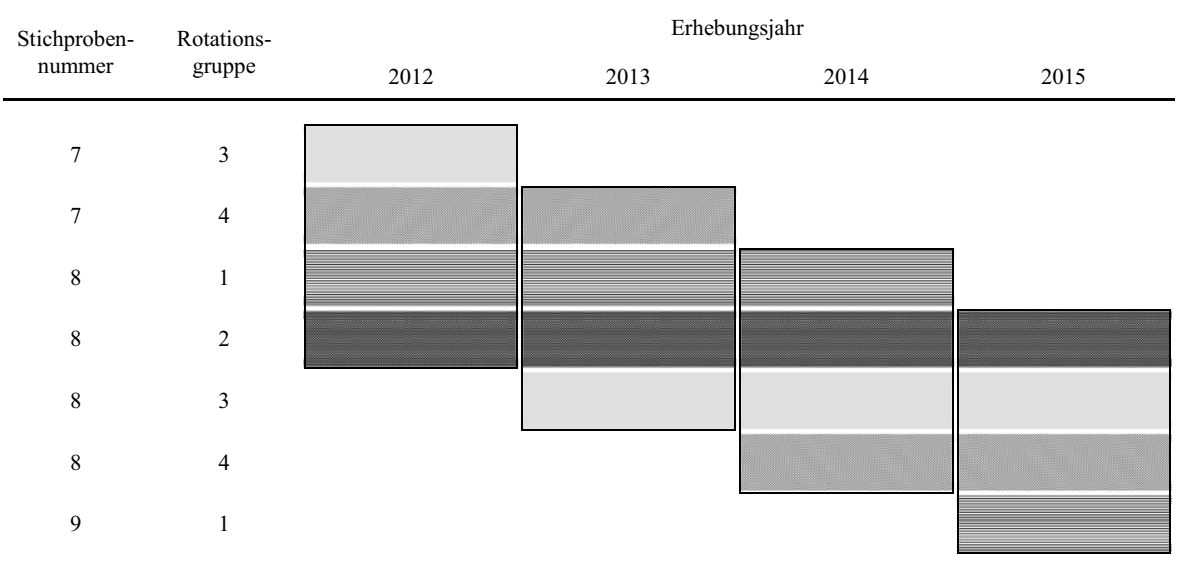

Abb. 1 Rotation im Mikrozensus (Quelle: Herter-Eschweiler und Schimpl-Neimanns 2018, S. 2)

Eine grundlegende Voraussetzung für die Zusammenführung von Beobachtungsund Stichprobeneinheiten sind zeitlich konsistente Identifikatoren (Ordnungsnummern). Des Weiteren ist in Bezug auf die Substichprobenziehung ausschlaggebend, dass die partielle Rotation auf der Ebene der Auswahlbezirke stattfindet. Mit der Umstellung der Ziehung von 70\%-Substichproben des SUF von der Haushaltsauf die Auswahlbezirksebene innerhalb eines Rotationsviertels und mit der Bereitstellung längsschnittkonsistenter Ordnungsnummern können die Eigenschaft eines Rotationspanels ab dem SUF des MZ 2012 genutzt werden. ${ }^{1}$ Der MZ ab 2016 basiert auf einer neuen Stichprobe und ab 2020 werden neben der EU-Arbeitskräfteerhebung weitere Haushaltsstatistiken in den MZ integriert (Bihler und Zimmermann 2016), sodass sich die hier beschriebenen Möglichkeiten auf die Daten 2012 bis 2015 beziehen.

Die Ziehung der Substichprobe orientiert sich eng am Stichprobenplan des MZ (siehe zur Kurzbeschreibung der Stichprobeneigenschaften Tab. 5 im Anhang). Informationen zur Klumpung liegen mit der Auswahlbezirksnummer vor. In Verbindung mit dem Stichprobenumfang des vollen MZ von 1\% der Auswahleinheiten wird als Ziehungswahrscheinlichkeit eines Auswahlbezirks des SUF aus der Menge aller Primäreinheiten in der Population 0,7\% angenommen.

In Bezug auf die bei der Schätzung von Querschnittsergebnissen verwendeten Schichtungsmerkmale ist zu beachten, dass das Berichtsquartal nicht die bei der Auswahl vorgesehenen, sondern die Quartale der realisierten Interviews enthält. Infolge teilweise verspäteter Antworten kann das Berichtsquartal in einem Auswahlbezirk variieren. Enthält die Schichtdefinition das Berichtsquartal, kann es bei der Analyse von Nettoveränderungen zu Schichtwechseln kommen. Laut Stichprobendesign gibt es keine unterjährige Rotation und unter Verwendung des Quartals gemäß Auswahl-

\footnotetext{
1 Siehe zu Möglichkeiten der Längsschnitt- und Panelauswertung Herberger (1973); Koller und Herberger (1960); Linke (1969); Statistisches Bundesamt o.J.; Forschungsdatenzentren der Statistischen Ämter des Bundes und der Länder (2018); Herter-Eschweiler und Schimpl-Neimanns (2018).
} 
plan wären Schichtwechsel ausgeschlossen (Afentakis und Bihler 2005, S. 1040; Statistisches Bundesamt 2013, S. 13). Tendenziell erhöhen Schichtwechsel die Varianz der Nettoveränderung. Ich schlage deshalb vor, bei der Schätzung von Nettoveränderungen für Jahresdurchschnitte auf das Berichtsquartal zur Abgrenzung von Schichten zu verzichten. ${ }^{2}$ Verwendet man zur Schichtabgrenzung das Bundesland und die Gebäudegrößenklasse (ohne Differenzierung nach dem Aktualisierungsjahr bei Neubauschichten) ergeben sich 79 Gruppen.

Die Hochrechnungsfaktoren rechnen auf 1000 der Population nach den Ergebnissen der laufenden Bevölkerungsfortschreibung mit dem Stand der Aktualisierung durch den Zensus 2011 hoch. Aufgrund der Auskunftspflicht ist der Anteil ausgefallener Haushalte sehr klein. Im MZ erfolgt die Kompensation der Ausfälle im Rahmen der Hochrechnung und Anpassung an bekannte Populationsverteilungen mittels verallgemeinerter Regressionsschätzung (siehe für Details Afentakis und Bihler 2005). Im Unterschied zum SUF bis 2011 sind nicht die Hochrechnungsfaktoren des MZ enthalten, sondern poststratifizierte Gewichtungsvariablen, die eine verbesserte Anpassung an die veröffentlichten Ergebnisse gewährleisten sollen. Zu diesem Zweck wurden die MZ-Hochrechnungsfaktoren mit einem ,Soll-durch-Ist“" Faktor multipliziert, der pro Anpassungsklasse das Verhältnis der Soll-Zahlen des MZ zu den Ist-Zahlen der Substichprobe bildet. Gewichtet wurde jeweils mit den Original Hochrechnungsfaktoren des MZ.

\section{Methode}

Für designbasierte Varianzschätzungen mit MZ-Querschnittsdaten liegen bereits Beschreibungen und Beispiele vor (Afentakis und Bihler 2005; Rendtel und SchimplNeimanns 2001; Schimpl-Neimanns 2011). Die folgende Darstellung kann deshalb kurz gehalten werden.

Obwohl die Auswahl der Primäreinheiten des SUF ab 2012 als Ergebnis eines 2-phasigen Ziehungsverfahrens betrachtet werden kann, genügt aufgrund des kleinen Auswahlsatzes des MZ die Annahme einer einstufigen Auswahl (Särndal et al. 1997, S. $139 \mathrm{ff}$.$) .$

Bei abhängigen Stichproben ist die Varianz der Differenz eines Schätzers $\widehat{\Delta}=$ $\widehat{\theta}_{2}-\widehat{\theta}_{1}$ allgemein $V(\widehat{\Delta})=V\left(\widehat{\theta}_{1}\right)+V\left(\widehat{\theta}_{2}\right)-2 \operatorname{Cov}\left(\widehat{\theta}_{1}, \widehat{\theta}_{2}\right)$. Je höher die Überlappung der Stichproben und je höher die jährlichen Indikatoren positiv korrelieren, desto geringer ist die Varianz der geschätzten Veränderung im Vergleich zu unabhängigen Stichproben. ${ }^{3}$ Im Folgenden werden zunächst die Schätzung für

\footnotetext{
${ }^{2}$ In dieser Hinsicht wäre eine Bereitstellung des nach dem Stichprobenplan vorgesehenen Quartals im SUF wünschenswert.

${ }^{3}$ Manchmal werden Daten verschiedener Zeitpunkte kumuliert, um die Fallzahlen von kleinen Teilpopulationen zu erhöhen. Bei der Schätzung von Summen $\left(\widehat{\Sigma}=\widehat{\theta}_{1}+\widehat{\theta}_{2}\right)$ ist die Varianz: $\operatorname{Var}(\widehat{\Sigma})=$ $V\left(\widehat{\theta}_{1}\right)+V\left(\widehat{\theta}_{2}\right)+2 \operatorname{Cov}\left(\widehat{\theta}_{1}, \widehat{\theta}_{2}\right)$. Im Unterschied zu unabhängigen Stichproben vergrößert die Korrelation den Stichprobenfehler.
} 
Querschnittsdaten und anschließend die Schätzung für Nettoveränderungen dargestellt.

\subsection{Designbasierte Schätzung für Querschnittsdaten}

Häufig geht es bei Nettoveränderungen um die Schätzung der Differenz von Verhältniswerten zu verschiedenen Zeitpunkten. Dies trifft auch für die unten ausgewählten Indikatoren zu, sodass sich die folgende Darstellung darauf beschränkt.

$$
\widehat{\theta}=\frac{\widehat{Y}}{\widehat{Z}}=\frac{\sum_{h=1}^{L} \sum_{i=1}^{n_{h}} \sum_{j=1}^{m_{h i}} w_{h i j} y_{h i j}}{\sum_{h=1}^{L} \sum_{i=1}^{n_{h}} \sum_{j=1}^{m_{h i}} w_{h i j} z_{h i j}}
$$

Ausgangspunkt für die Schätzung eines Indikators sei eine jährliche Stichprobe $(s)$ mit Angaben zu den Merkmalen $y_{h i j}$ im Zähler und $z_{h i j}$ im Nenner für jede $j$-te Elementareinheit (Person) im $i$-ten Auswahlbezirk der Schicht $h$. Die Angaben sind jeweils mit dem Anpassungsgewicht $w_{h i j}$ gewichtet.

Im Allgemeinen können mit der Anpassungsgewichtung mit den Veröffentlichungen der Statistischen Ämter konsistente Ergebnisse erzielt werden. Die Gewichtung führt aber zu einer größeren Variabilität unabhängig vom Stichprobenplan. Es wird deshalb eine Regressionsschätzung vorgenommen. Die Verwendung von Anpassungsgewichten kann näherungsweise als Regressionsschätzung interpretiert werden (Särndal et al. 1997, S. $264 \mathrm{ff} .$, S. $294 \mathrm{ff}$.). Dabei wird angenommen, dass die bei der Poststratifikation verwendeten Anpassungs- bzw. Hilfsmerkmale $(x)$ einen statistischen Einfluss auf die interessierende Variable haben, der durch eine lineare Regression modelliert werden kann. Es wird die approximierte linearisierte Variable $v_{h i j}=\left(y_{h i j}-\widehat{R} z_{h i j}\right) / \widehat{Z}$ des Verhältniswertes als interessierende Variable in der Regression eingesetzt (Särndal et al. 1997, S. 176ff., 294 ff.; Deville 1999, S. 198). Die mit dem Korrekturfaktor gewichteten Residuen $u_{h i j}=g_{h i j}\left(v_{h i j}-\boldsymbol{x}_{h i j}^{\prime} \widehat{\mathbf{B}}\right)$ bilden die Hilfsvariablen für die Schätzung der Varianz. Da die Anpassungsgewichte in den Daten bereits vorliegen, kann der Korrekturfaktor als Verhältnis von Anpassungs- und Designgewicht direkt ermittelt werden. Aufgrund der Linearisierung des Verhältniswertes kann die Varianz nur approximativ (AV) geschätzt werden. Sie ist asymptotisch erwartungstreu.

$$
\operatorname{AV}(\widehat{\theta})=\sum_{h=1}^{L} n_{h} /\left(n_{h}-1\right) \sum_{i=1}^{n_{h}}\left(u_{h i}-\bar{u}_{h}\right)^{2}
$$

mit

$\begin{array}{ll}h & \text { Schicht } h=1, \ldots, L \\ i & \text { Primäreinh. Auswahlbez., PSU } i=1, \ldots, n_{h} \\ j & \text { Sekundäreinheit Person } j=1, \ldots, m_{h i} \\ y_{h i j}, z_{h i j} & \text { Analysevariablen } \\ w_{h i j} & \text { End-/Anpassungsgewicht }\end{array}$




$\begin{array}{ll}f_{h}=0,007 & \text { Auswahlsatz } \\ d_{h i j}=N_{h} / n_{h}=1 / f_{h} & \text { Designgewicht } \\ g_{h i j}=w_{h i j} / d_{h i j} & \text { Korrekturfaktor } \\ u_{h i}=\sum_{j i}^{m_{h i}} d_{h i j} u_{h i j} & \text { PSU-Gesamtwert } \\ \bar{u}_{h}=1 / n_{h} \sum_{i=1}^{n_{h}} u_{h i} & \text { Mittelwert der PSU-Gesamtwerte }\end{array}$

Die Schätzung für geschichtete einstufige Klumpenstichproben (2) entspricht im Wesentlichen den bisherigen Anwendungen für Querschnittsdaten des SUF mit zwei Abweichungen. Zur Vermeidung von Schichtwechseln wird bei der Schichtabgrenzung auf das Berichtsquartal verzichtet. Außerdem wird analog zur Schätzung der Kovarianz (siehe Abschn. 3.2) von einer einfachen Zufallsstichprobe der Auswahlbezirke ausgegangen.

\subsection{Schätzungen für Nettoveränderungen}

Frühere Ansätze (Kish 1965, S. 457-468; Statistisches Bundesamt 1960, S. 115) verwendeten für die Schätzung der Kovarianz die in beiden Stichproben vorhandenen Einheiten. Dies setzt bei Flächenstichproben voraus, dass sich räumlich mobile und nicht mobile Einheiten in ihren Eigenschaften nicht unterscheiden. Dagegen nutzt der Ansatz von Berger und Priam (2016) die gesamten Daten der Stichproben auf der Ebene der Auswahlbezirke. Es werden außerdem keine Informationen über Inklusionswahrscheinlichkeiten zweiter Ordnung benötigt, die für anonymisierte Daten in der Regel unbekannt sind. Die Schätzung der Kovarianz erfolgt auf der Basis der Kovarianz der Residuen einer multivariaten Regression mit Informationen zum Stichprobendesign. Das Verfahren wird bei Schätzungen mit Daten der Europäischen Gemeinschaftsstatistik über Einkommen und Lebensbedingungen (EU-SILC) eingesetzt und hat sich beim schwedischen Labour Force Survey bewährt. Einige Annahmen und Einschränkungen sind jedoch zu beachten (Berger und Priam 2016, S. 252; S. 267; Berger 2004).

Im Hinblick auf den kleinen Auswahlsatz des SUF dürfte die Annahme einer einfachen Zufallsstichprobe der Auswahlbezirke und somit vernachlässigbarer Endlichkeitskorrektur unproblematisch sein. Mit der Abgrenzung der Schicht mittels Bundesland und Gebäudegrößenklasse liegt keine zu stark differenzierte Schichtung vor. Des Weiteren ist durch das Rotationsdesign des MZ sichergestellt, dass der Umfang der überlappenden Stichproben nicht zufällig ist. Die Annahme eines Stichprobendesigns mit hoher Entropie trifft auf den MZ zu. Für das SUF sind wegen der Substichprobenziehung Einschränkungen anzunehmen.

Die abhängigen Variablen der multivariaten Regression sind die auf der Ebene der Auswahlbezirke ( $i$ ) summierten Residuen der Regression der linearisierten Variablen auf die Hilfsmerkmale der designbasierten Schätzungen für die Querschnittsdaten zum Zeitpunkt $t$ und die jeweiligen Subpopulationen: $\widehat{\tau}_{1 i}=\sum_{i \in s_{1}} u_{i j}, \widehat{\tau}_{2 i}=$ $\sum_{i \in s_{2}} u_{i j}$. Falls ein Auswahlbezirk zu einem Zeitpunkt nicht vertreten ist, werden die Werte der abhängigen Variablen auf 0 gesetzt.

Die Kovariaten sind vollständige Interaktionen der Informationen zum Stichprobendesign und Rotationsdesign, d.h. der Schichtung und der Erhebungszeitpunkte. Dabei ist $z_{h 1 i}=1$, falls der $i$-te Auswahlbezirk zum Zeitpunkt $t=1$ zur $h$-ten 
Schicht gehört, und sonst 0 . Analog gilt $z_{h 2 i}=1$ für den Zeitpunkt $t=2$. Das Modell enthält keine Regressionskonstante (Berger und Priam 2016, S. 255):

$$
\left(\begin{array}{l}
\widehat{\tau}_{1 i} \\
\widehat{\tau}_{2 i}
\end{array}\right)=\left(\begin{array}{l}
\beta_{1}^{(1)} z_{h 1 i}+\beta_{2}^{(1)} z_{h 2 i}+\beta_{12}^{(1)} z_{h 1 i} z_{h 2 i} \\
\beta_{1}^{(2)} z_{h 1 i}+\beta_{2}^{(2)} z_{h 2 i}+\beta_{12}^{(2)} z_{h 1 i} z_{h 2 i}
\end{array}\right)+\left(\begin{array}{l}
\varepsilon_{1 i} \\
\varepsilon_{2 i}
\end{array}\right)
$$

Die Korrelation der Residuen der multivariaten Regression $\rho_{\widehat{\Sigma}}\left(\widehat{\theta}_{1}, \widehat{\theta}_{2}\right)$ wird als Schätzung der Korrelation der Schätzer der Querschnittsdaten verwendet. Die Korrelation der Residuen bezieht sich auf die Annahme einer einfachen Zufallsstichprobe ohne Hochrechnung. Gesucht ist aber die designbasierte Varianz mit Hochrechnung. Hierfür werden in $V(\widehat{\Delta})$ die für die Querschnittsdaten vorliegenden designbasierten Standardfehler $V\left(\widehat{\theta}_{t}\right)$ und die Korrelationen $\rho_{\widehat{\Sigma}}\left(\widehat{\theta}_{1}, \widehat{\theta}_{2}\right)$ aus der Kovarianzmatrix der Residuen eingesetzt. Allgemein besteht zwischen Korrelation und Kovarianz die Beziehung $\rho\left(\widehat{\theta}_{1}, \widehat{\theta}_{2}\right)=\operatorname{Cov}\left(\widehat{\theta}_{1}, \widehat{\theta}_{2}\right) /\left(V\left(\widehat{\theta}_{1}\right)^{-1 / 2} V\left(\widehat{\theta}_{1}\right)^{-1 / 2}\right)$. Die designbasierte Kovarianz $V\left(\widehat{\theta}_{2}, \widehat{\theta}_{1}\right)$ ergibt sich somit durch die Multiplikation der Korrelation $\rho_{\widehat{\Sigma}}\left(\widehat{\theta}_{1}, \widehat{\theta}_{2}\right)$ mit den designbasierten Standardfehlern von $V\left(\widehat{\theta}_{1}\right)$ und $V\left(\widehat{\theta}_{2}\right)$. Die Varianz der Differenz eines Schätzers $\widehat{\Delta}=\widehat{\theta}_{2}-\widehat{\theta}_{1}$ ist

$$
V(\widehat{\Delta})=V\left(\widehat{\theta}_{2}\right)+V\left(\widehat{\theta}_{1}\right)-2 \sqrt{V\left(\widehat{\theta}_{2}\right)} \sqrt{V\left(\widehat{\theta}_{1}\right)} \rho_{\widehat{\Sigma}}\left(\widehat{\theta}_{1}, \widehat{\theta}_{2}\right) .
$$

\section{Ergebnisse}

Am Beispiel von 4 Indikatoren werden im Folgenden Nettoveränderungen für die MZ-Erhebungen 2012 und 2013 geschätzt. Es wird untersucht, ob Veränderungen der Indikatoren als zufällige Schwankungen der Stichproben oder statistisch signifikant zu bewerten sind. Die Beispiele greifen in der Wissenschaft wie auch in der Öffentlichkeit thematisierte Fragen auf. Sie orientieren sich an der Sozialberichterstattung der amtlichen Statistik. Dort finden sich zahlreiche Beispiele einer nach Subpopulationen differenzierten Sozialberichterstattung unter anderem in den sogenannten Langen Reihen der Fachserien des Statistischen Bundesamtes, auf den Webseiten zur Sozialberichterstattung der Statistischen Ämter des Bundes und der Länder (2017) oder im Armuts- und Reichtumsbericht der Bundesregierung (BMAS 2017). Vergleichbare Ergebnisse zu Standardabweichungen oder Konfidenzintervallen für die hier betrachteten Indikatoren liegen leider nicht vor.

Der Indikator Erwerbstätigenquote für 60- bis 64-Jährige ist definiert als Anteil der Erwerbstätigen im Alter von 60 bis 64 Jahren an der gleichaltrigen Bevölkerung. Die Erwerbstätigenquote für diese Altersgruppe knüpft an die Themen der Erhöhung des effektiven Renteneintrittsalters und des alterungsbedingten Arbeitskräftemangels an. Die Erwerbstätigenquote für 20- bis 64-Jährige ist ein Leitindikator der Europa 2020-Strategie der Europäischen Kommission (2018). 
Tab. 1 Erwerbstätigenquote für 60- bis 64-Jährige: Jahresdurchschnitte, Nettoveränderung und Standardfehler

\begin{tabular}{lllll}
\hline Jahr & Anteil (\%) & SE (\%) & KI 2,5\% & KI 97,5\% \\
\hline 2013 & 49,7 & 0,2338 & 49,3 & 50,2 \\
2012 & 46,7 & 0,2292 & 46,3 & 47,2 \\
$\Delta$ 2013-2012 & 3,0 & 0,2421 & 2,51 & 3,46 \\
Annahme: Unabh & - & 0,3275 & 2,35 & 3,63 \\
\hline
\end{tabular}

Quelle: FDZ der Statistischen Ämter des Bundes und der Länder, Mikrozensus (2012, 2013), eigene Berechnungen. Bevölkerung am Ort der Hauptwohnung, hochgerechnete Werte

SE Standardfehler, KI 95\%-Konfidenzintervall, Annahme: Unabh. Ohne Berücksichtigung der Kovarianz unter Annahme statistischer Unabhängigkeit der Schätzer zwischen den Erhebungszeitpunkten

Tab. 2 Teilzeitquote für abhängig Beschäftigte im Alter von 15 bis 64 Jahren: Jahresdurchschnitte, Nettoveränderung und Standardfehler

\begin{tabular}{lllll}
\hline Jahr & Anteil (\%) & SE (\%) & KI 2,5\% & KI 97,5\% \\
\hline 2013 & 27,0 & 0,1007 & 26,9 & 27,2 \\
2012 & 26,5 & 0,0985 & 26,3 & 26,7 \\
$\Delta$ 2013-2012 & 0,5 & 0,1036 & 0,31 & 0,72 \\
Annahme: Unabh & - & 0,1408 & 0,24 & 0,79 \\
\hline
\end{tabular}

Quelle: FDZ der Statistischen Ämter des Bundes und der Länder, Mikrozensus (2012, 2013), eigene Berechnungen. Bevölkerung am Ort der Hauptwohnung, hochgerechnete Werte

SE Standardfehler, KI Konfidenzintervall, Annahme: Unabh. Ohne Berücksichtigung der Kovarianz unter Annahme statistischer Unabhängigkeit der Schätzer zwischen den Erhebungszeitpunkten

Die Befristungsquote erfasst den Anteil befristeter Arbeitsverträge. Befristete Beschäftigungen zählen zu den atypischen Beschäftigungen. Sie haben in den letzten Dekaden im Vergleich zu sogenannten Normalarbeitsverhältnissen insgesamt zugenommen (BMAS 2017, S. $79 \mathrm{ff}$.). Bei guter Konjunktur sind Befristungen jedoch tendenziell rückläufig. In Anlehnung an die Abgrenzung des Statistischen Bundesamtes (2017, S. 40) werden nur abhängig Beschäftigte ab 25 Jahren betrachtet.

Die Teilzeitquote bezieht sich auf abhängig Beschäftigte in Teilzeitarbeit im Alter von 15 bis 64 Jahren unter allen gleichaltrigen abhängig Beschäftigten. Aus Sicht der Erwerbstätigen wird häufig Teilzeitarbeit gewählt, um Aufgaben in den Bereichen Familie und Beruf besser vereinbaren zu können. Insgesamt haben Teilzeitbeschäftigungen im Zeitverlauf zugenommen. Im MZ 2013 wurde die Zuordnung der Selbsteinschätzung der Befragten zur Voll- oder Teilzeittätigkeit geändert. Um vergleichbare Ergebnisse zu gewährleisten, wurde Teilzeitarbeit für 1 bis einschließlich 31 normalerweise geleisteten Wochenarbeitsstunden definiert.

Der Leitindikator der Strategie Europa 2020 Frühzeitige Schul- und Ausbildungsabgänger zeigt den Anteil von 18- bis 24-Jährigen, die höchstens einen Haupt- oder Realschulabschluss haben, zum Zeitpunkt der Befragung keine schulische oder berufliche Ausbildung absolvieren oder an Weiterbildungsmaßnahmen teilnehmen, an der gleichaltrigen Bevölkerung. Der Indikator beschreibt damit eine Gruppe, die auf dem Arbeitsmarkt mit überdurchschnittlichen Schwierigkeiten konfrontiert ist. Das Europa-2020-Ziel von höchstens 10\% wurde 2013 in Deutschland erstmals erreicht.

Die folgenden Tabellen enthalten sowohl für die Querschnittsdaten als auch die Nettoveränderungen die mit den oben dargestellten Verfahren geschätzten Quo- 
Tab. 3 Anteil früher Schulabgänger im Alter von 18 bis 24 Jahren: Jahresdurchschnitte, Nettoveränderung und Standardfehler

\begin{tabular}{lllll}
\hline Jahr & Anteil (\%) & SE (\%) & KI 2,5\% & KI 97,5\% \\
\hline 2013 & 9,9 & 0,1863 & 9,5 & 10,2 \\
2012 & 10,4 & 0,1893 & 10,0 & 10,8 \\
$\Delta 2013-2012$ & $-0,5$ & 0,2148 & $-0,94$ & $-0,10$ \\
Annahme: Unabh & - & 0,2657 & $-1,04$ & $-0,00$ \\
\hline
\end{tabular}

Quelle: FDZ der Statistischen Ämter des Bundes und der Länder, Mikrozensus (2012, 2013), eigene Berechnungen. Bevölkerung in Privathaushalten am Ort der Hauptwohnung (EU-Definition), hochgerechnete Werte

SE Standardfehler, KI Konfidenzintervall, Annahme: Unabh. Ohne Berücksichtigung der Kovarianz unter Annahme statistischer Unabhängigkeit der Schätzer zwischen den Erhebungszeitpunkten

Tab. 4 Befristungsquote für abhängig Beschäftigte ab 25 Jahren: Jahresdurchschnitte, Nettoveränderung und Standardfehler

\begin{tabular}{lllll}
\hline Jahr & Anteil (\%) & SE (\%) & KI 2,5\% & KI 97,5\% \\
\hline 2013 & 8,3 & 0,0755 & 8,2 & 8,5 \\
2012 & 8,5 & 0,0763 & 8,4 & 8,7 \\
$\Delta$ 2013-2012 & $-0,2$ & 0,0894 & $-0,37$ & $-0,02$ \\
Annahme: Unabh & - & 0,1074 & $-0,41$ & 0,01 \\
\hline
\end{tabular}

Quelle: FDZ der Statistischen Ämter des Bundes und der Länder, Mikrozensus (2012, 2013), eigene Berechnungen. Bevölkerung am Ort der Hauptwohnung, hochgerechnete Werte

SE Standardfehler, KI Konfidenzintervall, Annahme: Unabh. Ohne Berücksichtigung der Kovarianz unter Annahme statistischer Unabhängigkeit der Schätzer zwischen den Erhebungszeitpunkten

ten, designbasierte Standardfehler sowie 95\%-Konfidenzintervalle. In der jeweils letzten Zeile werden Schätzungen unter Annahme der statistischen Unabhängigkeit der Teilstichproben berichtet, die bisher aufgrund fehlender längsschnittkonsistenter Ordnungsnummern getroffen werden musste. Der Vergleich mit den Ergebnissen für Nettoveränderungen unter Berücksichtigung der Kovarianz zeigt, welche Überschätzungen der Standardfehler bzw. der Konfidenzintervalle entstehen, wenn die Kovarianz nicht berücksichtigt wird.

Von 2012 bis 2013 ist die Erwerbstätigenquote für 60- bis 64-Jährige um 3 Prozentpunkte gestiegen (Tab. 1). Der Standardfehler beträgt 0,24\% und das dazugehörige $95 \%$-Konfidenzintervall 2,5 bis 3,5\%. Es enthält nicht den Wert null, sodass die Veränderung als statistisch signifikant gilt. Dieser Schluss würde auch ohne Berücksichtigung der Kovarianz gezogen, wenngleich mit einem um 35 Prozentpunkte höheren Standardfehler als bei korrekter Berücksichtigung der Kovarianz der Schätzer.

In ähnlicher Weise trifft dies auch auf den Anstieg der Teilzeitquote um 0,5 Prozentpunkte (Tab. 2) und den Rückgang des Anteils früher Schulabgänger um 0,5 Prozentpunkte (Tab. 3) zu. Allerdings beträgt in Tab. 3 die Überschätzung des Standardfehlers bei Annahme statistischer Unabhängigkeit rund 24 Prozentpunkte im Vergleich zur Berücksichtigung der Kovarianz.

Im Unterschied dazu zeigt sich beim Rückgang der Befristungsquote um 0,2 Prozentpunkte (Tab. 4) ohne Berücksichtigung der Kovarianz keine statistisch signifikante Veränderung; das 95\%-Konfidenzintervall enthält den Wert null. Dagegen 
wird bei Berücksichtigung der Kovarianz eine signifikante Veränderung festgestellt. Wie dieses Beispiel zeigt, kann die nicht zutreffende Annahme statistischer Unabhängigkeit durchaus zu anderen Ergebnissen als die korrekte Varianzschätzung führen.

\section{Schluss}

Mit den ab dem MZ 2012 zur Verfügung stehenden Daten können Forscher nach der Umstellung der Substichprobenziehung und mit der Bereitstellung längsschnittkonsistenter Ordnungsnummern designbasierte Varianzschätzungen von Nettoveränderungen vornehmen. Gegenüber den bisherigen Schätzungen für Querschnittsdaten ist für die Varianzschätzung von Nettoveränderungen nach dem Verfahren von Berger und Priam (2016) kein großer Mehraufwand erforderlich.

Am Beispiel von 4 Indikatoren zeigten sich erhebliche Unterschiede, ob die durch die partielle Rotation entstehende Kovarianz der Schätzungen statistisch angemessen berücksichtigt wird oder nicht. Nimmt man fälschlicherweise statistische Unabhängigkeit der Stichproben an, ist der Standardfehler der Nettoveränderung um 20 bis 35 Prozentpunkte größer als bei Berücksichtigung der Kovarianz der Schätzer. Zudem zeigte sich, dass die Annahme statistischer Unabhängigkeit durchaus zu anderen Einschätzungen der statistischen Signifikanz führen kann. Zusammenfassend kann deshalb festgehalten werden, dass die ab 2012 geschaffenen Möglichkeiten es der Wissenschaft erlauben, die hohe Präzision der Schätzergebnisse von Nettoveränderungen effizient auszuschöpfen.

Danksagung Den Herausgebern und anonymen Gutachtern danke ich für konstruktive und wichtige Änderungsvorschläge. Für Anmerkungen zu einer früheren Version des Manuskripts danke ich Wolf Bihler, Robert Herter-Eschweiler, Heike Wirth und Stefan Zins.

Open Access Dieser Artikel wird unter der Creative Commons Namensnennung 4.0 International Lizenz (http://creativecommons.org/licenses/by/4.0/deed.de) veröffentlicht, welche die Nutzung, Vervielfältigung, Bearbeitung, Verbreitung und Wiedergabe in jeglichem Medium und Format erlaubt, sofern Sie den/die ursprünglichen Autor(en) und die Quelle ordnungsgemäß nennen, einen Link zur Creative Commons Lizenz beifügen und angeben, ob Änderungen vorgenommen wurden. 


\section{Anhang}

Tab. 5 Kurzbeschreibung des Mikrozensus und der Mikrozensus Scientific-Use-Files ab 2012

\begin{tabular}{ll}
\hline Stichprobeneigenschaften & Beschreibung \\
\hline Mikrozensus (2012) & \\
Grundgesamtheit & $\begin{array}{l}\text { Wohnberechtigte Bevölkerung in Privathaushalten und Gemein- } \\
\text { schaftsunterkünften }\end{array}$ \\
& Jeweils letzte Kalenderwoche vor der Befragung (gleitende Be- \\
richtswoche). & Aufgrund von Problemen der Erreichbarkeit der zu befragenden \\
& Haushalte finden die gemäß Stichprobenplan in einem bestimmten \\
& Zeitraum geplanten Interviews teilweise erst im folgenden Quartal \\
& oder Folgejahr (sog. Jahresüberhänge) statt. Verspätet realisierte \\
& Interviews werden wie reguläre Interviews zum Berichtszeitraum \\
& behandelt
\end{tabular}

Erhebungseinheiten

Auswahlgrundlage

Auswahlverfahren

Schichtung

Auswahleinheiten

Partielle Rotation

Auswahltechnik

Auswahlsatz

Stichprobenumfang

Hochrechnung
Haushalte, Personen

Alte Bundesländer: Ergebnisse der Volkszählung 1987; Neue Bundesländer: Bevölkerungsregister „Statistik“ 1991; Aktualisierung der Stichprobe unter Berücksichtigung der Neubautätigkeit

Einstufig geschichtete Klumpenstichprobe

Bundesland, Regionalschicht, Gebäudeschicht (Gebäudegrößenklasse)

Auswahlbezirke: Klumpen von i.d.R. zusammenliegenden Gebäuden bzw. Gebäudeteilen. Je nach Gebäudegröße bzw. -typ wurde eine unterschiedliche durchschnittliche Zahl von Wohnungen bzw. Personen je Auswahlbezirk vorgesehen

Ein Auswahlbezirk verbleibt vier Jahre in der Stichprobe. In jedem Jahr scheidet ein Viertel der Auswahlbezirke aus und ein Viertel wird neu aufgenommen. Aus einem Auswahlbezirk wegziehende Haushalte und Personen werden nicht weiterbefragt, sondern durch zuziehende Haushalte und Personen ersetzt (Prinzip der Flächenstichprobe)

Die Grundauswahl entspricht zusammenfassend einer uneingeschränkten Zufallsauswahl. Die Aktualisierung/Neubauauswahl entspricht einer systematischen Auswahl mit Zufallsstart $1 \%$

49.200 Auswahlbezirke mit Befragungshaushalten, 688.900 Befragte

Zweistufiges Verfahren: 1. Kompensation der bekannten Ausfälle auf Haushaltsebene (Anteil ausgefallener Haushalte: 2,4\%; ohne Jahresüberhänge: 1,1\%). 2. Anpassung der Stichprobenergebnisse an Eckzahlen aus der Laufenden Bevölkerungsfortschreibung mit dem Stand der Aktualisierung durch den Zensus 2011 auf der Ebene regionaler Anpassungsschichten. Quartalsweise Hochrechnung auf unterschiedlichen regionalen Ebenen mittels verallgemeinerter Regressionsschätzung. Alle Personen in einem Haushalt erhalten den gleichen Gewichtungsfaktor 
Tab. 5 (Fortsetzung)

\begin{tabular}{|c|c|}
\hline Stichprobeneigenschaften & Beschreibung \\
\hline \multicolumn{2}{|l|}{ Scientific-Use-File ab 2012} \\
\hline Auswahlverfahren & $\begin{array}{l}\text { Ziehung einer } 70 \% \text {-Substichprobe von Auswahlbezirken aus dem } \\
\text { vollen MZ }\end{array}$ \\
\hline Schichtung durch Anordnung & $\begin{array}{l}\text { Sortierung der Auswahlbezirke nach Bundesland, Größenklas- } \\
\text { se (Dezile) der Auswahlbezirke, Kennung über Grundauswahl/ } \\
\text { Aktualisierung der Auswahlbezirke, Kennung über den Einbezug } \\
\text { eines Auswahlbezirks in das Ad-hoc-Modul, Regierungsbezirk, } \\
\text { Regionale Anpassungsschicht, Regionale Schicht, Regionale Un- } \\
\text { tergruppe, Gemeindegrößenklasse und Nummer des Auswahlbe- } \\
\text { zirks }\end{array}$ \\
\hline Auswahltechnik & $\begin{array}{l}\text { Jeweils zehn in der Reihenfolge der Sortierung aufeinanderfolgen- } \\
\text { de Auswahlbezirke bilden eine Schicht. Bei Sortierwechsel von } \\
\text { Bundesland und Größenklassen (Dezile) der Auswahlbezirke sowie } \\
\text { bei der letzten Schicht sind unvollständige Schichten mit weniger } \\
\text { als } 10 \text { Auswahlbezirken zulässig. Ziehen aller Auswahlbezirke, } \\
\text { deren letzte Platzziffer einer von } 7 \text { ganzzahligen Zufallszahlen im } \\
\text { Intervall } \alpha \text { mit ( } 1 \leq \alpha \leq 10, \alpha \in \mathbb{N} \text { ) entspricht, d.h. Übernahme } \\
\text { von } 70 \% \text { der Auswahlbezirke des vollen MZ in die Substichpro- } \\
\text { be. Die Ziehungswahrscheinlichkeit eines Auswahlbezirks des } \\
\text { SUF aus der Menge aller Primäreinheiten der Population beträgt } \\
\text { demnach } 0,7 \%\end{array}$ \\
\hline Substichprobenumfang & $\begin{array}{l}\text { Rund } 34.200 \text { Auswahlbezirke mit Befragungshaushalten, } 482.100 \\
\text { Befragte }\end{array}$ \\
\hline Hochrechnung & $\begin{array}{l}\text { Die Hochrechnungsfaktoren des vollen MZ, multipliziert mit dem } \\
\text { Kehrwert der Auswahlwahrscheinlichkeit des SUF (100/70), wur- } \\
\text { den nachträglich an hochgerechnete Verteilungen angepasst. Die } \\
\text { Anpassungsmerkmale (Zahl der Kategorien) sind pro Bundes- } \\
\text { land (16): Geschlecht (2), Altersgruppe (4) und Erwerbsstatus (2). } \\
\text { Empirisch ergeben sich } 224 \text { Anpassungsklassen. } 2012 \text { erfolgte die } \\
\text { Poststratifikation ohne Beachtung des Haushaltszusammenhangs, } \\
2013 \text { sind die Hochrechnungsfaktoren für die Haushaltsmitglieder } \\
\text { eines Haushalts gleich }\end{array}$ \\
\hline
\end{tabular}

Quelle: Statistisches Bundesamt (2012); Statistisches Bundesamt (2013); Statistisches Bundesamt und GESIS (2016); eigene Zusammenstellung

Hinweis des Verlags Der Verlag bleibt in Hinblick auf geografische Zuordnungen und Gebietsbezeichnungen in veröffentlichten Karten und Institutsadressen neutral.

\section{Literatur}

Afentakis A, Bihler W (2005) Das Hochrechnungsverfahren beim unterjährigen Mikrozensus ab 2005. Wirtsch Stat 10:1039-1048

Berger YG (2004) Variance estimation for measures of change in probability sampling. Can J Stat 32(4):451-467

Berger YG, Priam R (2016) A simple variance estimator of change for rotating repeated surveys: An application to the European union statistics on income and living conditions household surveys. J Royal Stat Soc Ser A 179:251-272

Bihler W, Zimmermann D (2016) Die neue Mikrozensusstichprobe ab 2016. https://www.destatis. de/DE/Publikationen/WirtschaftStatistik/2016/06/DieNeueMikrozensusstichprobe_062016.pdf? blob=publicationFile. Zugegriffen: 12. Dez. 2018 
Bundesministerium für Arbeit und Soziales (2017) Lebenslagen in Deutschland. Der Fünfte Armuts- und Reichtumsbericht der Bundesregierung. http://www.armuts-und-reichtumsbericht.de/SharedDocs/ Downloads/Berichte/5-arb-langfassung.pdf?_blob=publicationFile\&v=6. Zugegriffen: 12. Dez. 2018

Deville JC (1999) Variance estimation for complex statistics and estimators: Linearization and residual techniques. Surv Methodol 25(2):193-203

Europäische Kommission (2018) Leitindikatoren: Scoreboard. http://ec.europa.eu/eurostat/de/web/europe2020-indicators/europe-2020-strategy/headline-indicators-scoreboard. Zugegriffen: 12. Dez. 2018

Forschungsdatenzentren der Statistischen Ämter des Bundes und der Länder (2018) Datenangebot । Mikrozensus-Panel. https://www.forschungsdatenzentrum.de/de/haushalte/mikrozensus-panel. Zugegriffen: 12. Dez. 2018

Herberger L (1973) Praktische Erfahrungen mit Verlaufsstatistiken. All Stat Arch 57:54-76

Herter-Eschweiler R, Schimpl-Neimanns B (2018) Möglichkeiten der Verknüpfung von Mikrozensus-Querschnitterhebungen ab 2012 zu Panels. https:/www.gesis.org/missy/files/documents/MZ/ panelbildung_suf2012.pdf. Zugegriffen: 12. Dez. 2018

Kish L (1965) Survey sampling. Wiley, New York

Koller S, Herberger L (1960) Der Mikrozensus. All Stat Arch 44(3):205-254

Linke W (1969) Umschichtung der Erwerbsbevölkerung. Ergebnisse der Mikrozensusbefragungen 1966 und 1967. Wirtsch Stat 9:505-508

Rendtel U, Schimpl-Neimanns B (2001) Die Berechnung der Varianz von Populationsschätzern im Scientific Use File des Mikrozensus ab 1996. https://www.gesis.org/fileadmin/upload/forschung/ publikationen/zeitschriften/zuma_nachrichten/zn_48.pdf. Zugegriffen: 12. Dez. 2018

Särndal CE, Swensson B, Wretman J (1997) Model assisted survey sampling, 4. Aufl. Springer, New York

Schimpl-Neimanns B (2011) Schätzung des Stichprobenfehlers in Mikrozensus Scientific Use Files ab 2005. AStA Wirtsch Sozialstat Arch 5(1):19-38. https://doi.org/10.1007/s11943-011-0092-4

Statistische Ämter des Bundes und der Länder (2017) Sozialberichterstattung der amtlichen Statistik. http://www.amtliche-sozialberichterstattung.de. Zugegriffen: 12. Dez. 2018

Statistisches Bundesamt (Hrsg) (1960) Stichproben in der Amtlichen Statistik. Kohlhammer, Stuttgart, Mainz

Statistisches Bundesamt (2012) Mikrozensus: Haushaltszahlen ab 2005. https://www.destatis.de/DE/ Methoden/MikrozensusHaushaltszahlen.pdf?_blob=publicationFile. Zugegriffen: 12. Dez. 2018

Statistisches Bundesamt (2013) Qualitätsbericht Mikrozensus 2012. https://www.destatis.de/DE/Publika tionen/Qualitaetsberichte/Bevoelkerung/Mikrozensus2012.pdf?_blob=publicationFile. Zugegriffen: 12. Dez. 2018

Statistisches Bundesamt (2017) Qualität der Arbeit. https://www.destatis.de/DE/Publikationen/Thematisch/ Arbeitsmarkt/Erwerbstaetige/BroschuereQualitaetArbeit0010015179004.pdf?_blob=publicationFile. Zugegriffen: 12. Dez. 2018

Statistisches Bundesamt (o. J.) Webseite Wissenschaftsforum - Methodenverbund „Aufbereitung und Bereitstellung des Mikrozensus als Panelstichprobe“. https://www.destatis.de/DE/Methoden/Methoden papiere/Mikrozensus/Mikrozensus.html. Zugegriffen: 12. Dez. 2018

Statistisches Bundesamt, GESIS (2016) Wichtige Informationen zur Nutzung des Mikrozensus Scientific Use Files 2012. http://www.gesis.org/missy/files/documents/MZ/readme/readme_suf2012.pdf. Zugegriffen: 12. Dez. 2018 